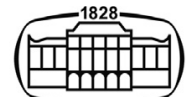

AKADÉMIAI KIADÓ

IMAGING

REVIEW ARTICLE

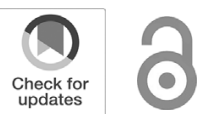

IMAGING 13 (2021) 2, 98-105 DOI: $10.1556 / 1647.2021 .00033$

(c) 2021 The Author(s)

*Corresponding author. Medical Imaging Centre, Semmelweis University, Korányi Sándor utca, 1083 Budapest, Hungary. Mobile: +3620 663 2485; Tel.: +36 20825 6889; fax: +3614586842 .

E-mail: maurovich-horvat.pal@med. semmelweis-univ.hu

\section{Quantitative plaque assessment by coronary computed tomography angiography: An up-to-date review}

\author{
NATASA JÁVORSZKY ${ }^{1}$, JUDIT SIMON ${ }^{1}$ and \\ PÁL MAUROVICH-HORVAT ${ }^{1,2 *}$ ๑
}

\author{
${ }^{1}$ MTA-SE Cardiovascular Imaging Research Group, Heart and Vascular Center, Semmelweis \\ University, Budapest, Hungary \\ ${ }^{2}$ Medical Imaging Centre, Semmelweis University, Budapest, Hungary
}

Received: December 28, 2020 • Accepted: May 11, 2021

\begin{abstract}
Coronary computed tomography angiography has an emerging role in the diagnostic workup of coronary artery disease. Due to its high sensitivity and negative predictive value, coronary computed tomography angiography can rule out obstructive coronary artery diseases and substitute invasive coronary angiography in many cases. In addition, coronary computed tomography angiography provides a unique information beyond stenosis grading as it can visualize atherosclerosis and quantify its extent. Qualitative and quantitative plaque assessment provides an incremental value in the prediction of future major adverse cardiac events. Moreover, determining adverse plaque features has a potential to identify advanced atherosclerosis and patients at increased risk of acute coronary syndrome. Nevertheless, challenges may emerge with the process of quantifying coronary plaques due to limited reproducibility, lack of automated, standardized and validated techniques. Therefore, reliable quantified data are scarce due to the various computed tomography scanners and software platforms and investigations with small sample sizes. Radiomics and machine learning-based image processing methods are relatively new in the field of cardiovascular plaque imaging. These techniques hold the promise to improve diagnostic performance, reproducibility and prognostic value of computed tomography based plaque assessment.
\end{abstract}

\section{KEYWORDS}

coronary plaque, radiomics, quantitative assessment, coronary CTA, machine learning, advanced plaque features

\section{Introduction}

Coronary computed tomography angiography (CTA) is an emerging tool in cardiovascular imaging. Due to its high sensitivity and negative predictive value, coronary CTA is a first line modality to rule out obstructive coronary artery disease (CAD) $[1,2]$. Patients with suspected coronary artery disease and low-to-intermediate pre-test probability can be referred to coronary CTA, whereas symptomatic, high-risk patients with high clinical likelihood of severe stenosis are referred functional imaging or direct to invasive coronary angiography $[1,2]$.

Coronary CTA not only enables the anatomical assessment of the coronary arteries, but it can quantify and characterize coronary plaques as well. Qualitative (Fig. 1) and quantitative (Fig. 2) plaque assessment are both feasible with coronary CTA. In current clinical practice, mostly qualitative methods are used for the evaluation of coronary CTA, while quantitative plaque assessment methods are in experimental stages. Thereby, clinical decisions are based on the localization and severity of visually assessed luminal diameter stenosis [2].

Due to their high spatial resolution, modern computed tomography scanners allow to quantify coronary plaques and determine plaque composition. Accurate plaque quantification 


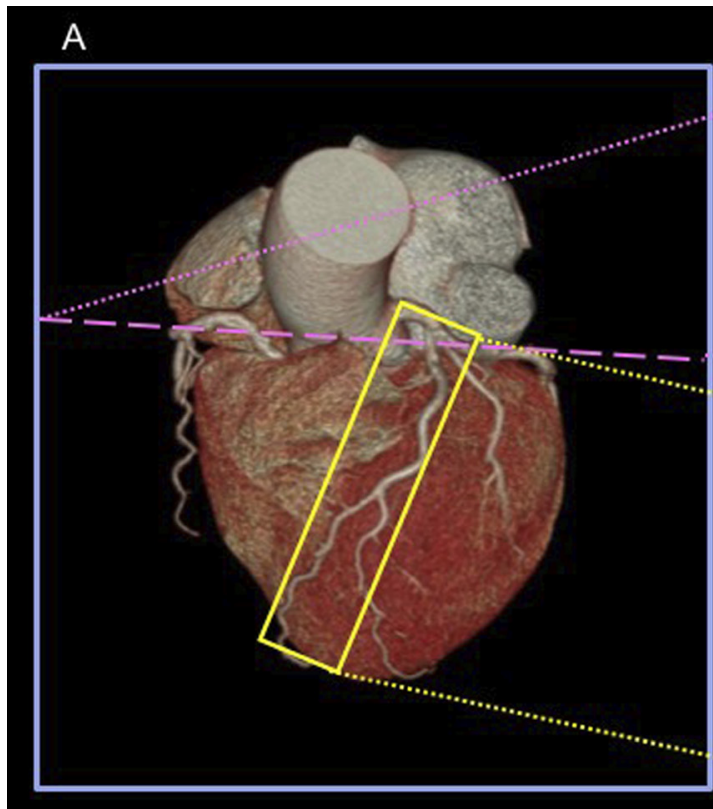

\section{B}
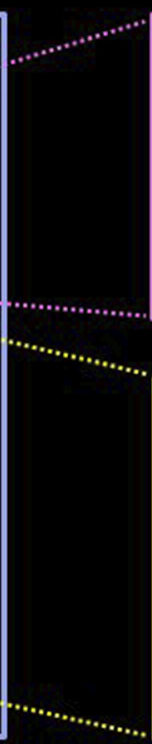

C
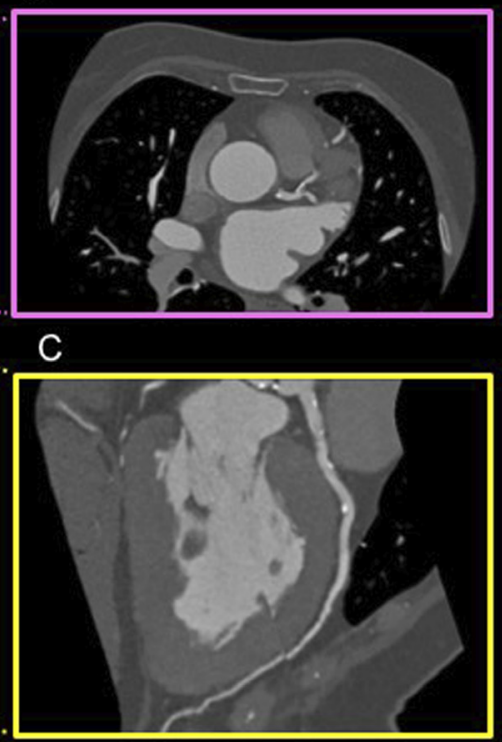

D

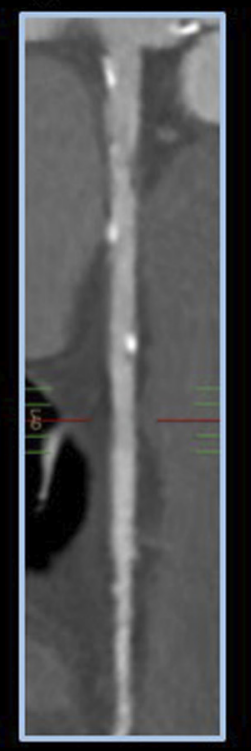

Fig. 1. Qualitative plaque assessment

Conventional qualitative assessment of CTA images (A) enables stenosis localization, stenosis grading and establishing calcium scoring. Cross-sectional image of the 3D CTA heart image (B); stretched multiplanar-reformatted image of the selected artery (C); straightened image of the artery (D)

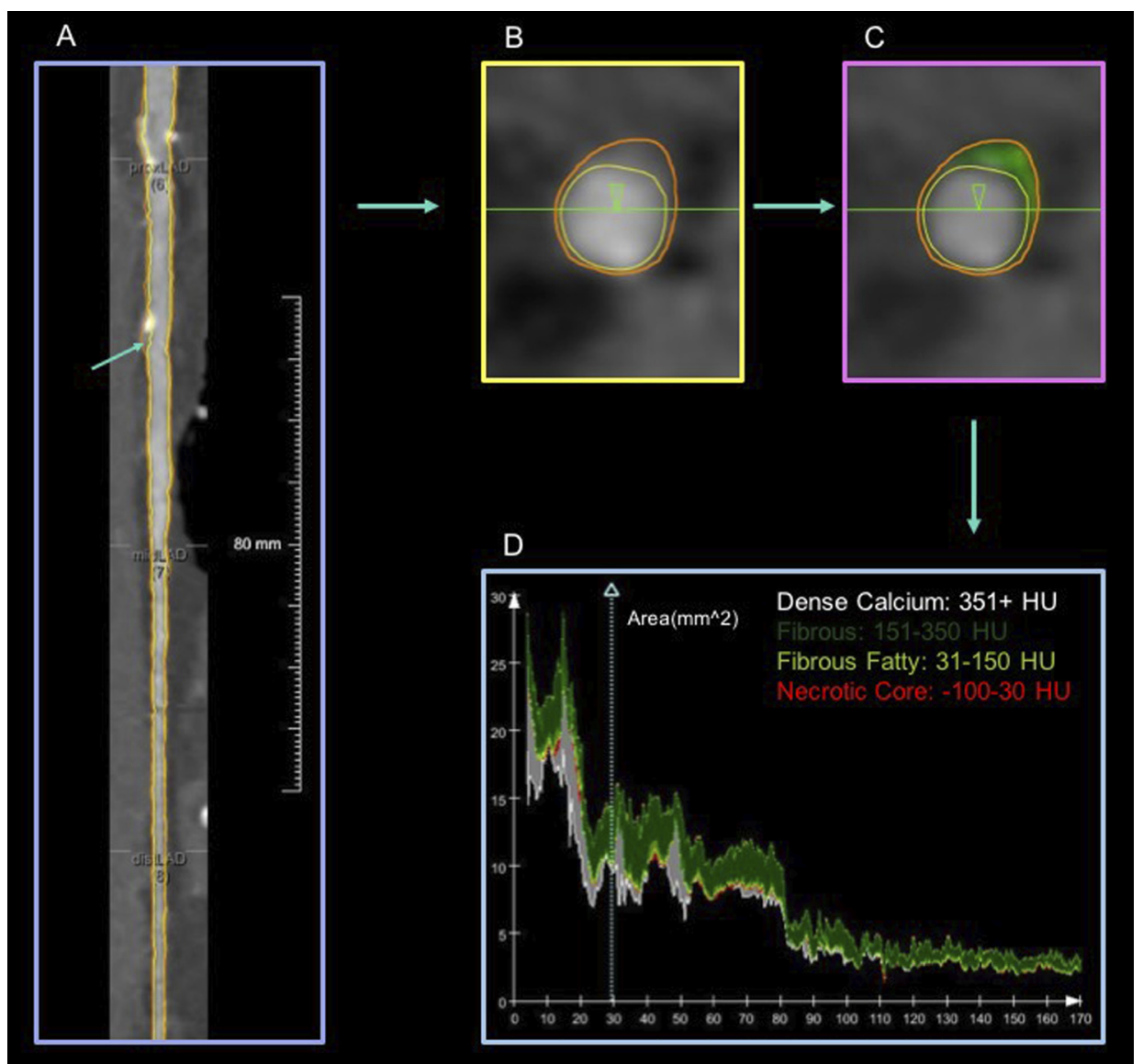

Fig. 2. Quantitative plaque assessment

Quantitative plaque reading (A) results in assessing plaque burden, area and volume (B) along with classifying the textural features determined by pixel attenuation values $(\mathrm{C}, \mathrm{D})$. Classification is based on predetermined cut-off values (D) 
and characterization are feasible with coronary CTA and calculated measurements correlate highly with intravascular ultrasound (IVUS) [3]. The ultimate goal of quantitative atherosclerotic plaque assessment is to gain more in-depth knowledge on the presence of CAD and improve risk stratification, potentially achieving a better and more appropriate treatment and eventually improving overall clinical outcome. By quantifying coronary plaques and determining adverse plaque features, we gain access to a wider range of information which may improve plaque characterization [4]. Use of radiomics and machine learning techniques can further improve the prognostic power of coronary CTA $[5,6]$. The application of radiomic technique based medical imaging in the field of cardiovascular research is a somewhat novel method of coronary plaque evaluation. Radiomics extracts hundreds of different parameters for each lesion from radiological images that can be further used to quantify morphological characteristics that are well beyond visual assessment [7]. In this article we review the prognostic value and methodological details of quantitative plaque assessment.

\section{The prognostic role of quantitative plaque assessment}

Quantitative plaque assessment considers several computercalculated factors such as plaque volume (PV), plaque burden (ratio of plaque volume to vessel volume, PB [8]), or plaque area. Moreover, this method provides information on plaque attenuation and remodeling. These characteristics have advantages compared to visual assessment of luminal diameter stenosis. In a prospectively designed study analyzing the results of the PARADIGM (Progression of AtheRosclerotic PlAque DetermIned by Computed TomoGraphic Angiography Imaging) registry involving 1,345 patients, the additional value of quantitative plaque assessment over qualitative coronary CTA evaluation methods proved to improve the prediction of rapid plaque progression and adverse clinical outcomes [9].

In another study, plaque quantification parameters such as $\mathrm{PV}, \mathrm{PB}$, plaque area, plaque attenuation and plaque remodeling were found to be significant predictors of acute coronary syndrome (ACS), while conventional coronary CTA parameters did not show significant difference between patients with and without ACS. When comparing the incremental prognostic values of the qualitative and quantitative plaque assessment techniques, quantitative plaque assessment had additive prognostic value over conventional CTA assessment and Framingham risk-score (FRS) (area under the curve $(\mathrm{AUC})=0.64$ for FRS + conventional coronary CTA assessment vs AUC $=0.79$ for FRS + conventional coronary CTA + quantitative plaque assessment $(P<0.05))[10]$.

Invasive measurement of fractional flow reserve (FFR) is considered as gold standard for the determination of hemodynamic significance of a coronary lesion. This is the merit of the CREDENCE (Computed Tomographic Evaluation of Atherosclerotic Determinants of Myocardial Ischemia) trial that it highlighted the diagnostic accuracy of coronary CTA. The analysis of coronary CTA assessment was found to provide superior vessel specific FFR estimation to myocardial perfusion imaging (AUC $=0.81$ and 0.67 , respectively $(P<0.001))[11]$. In prior studies, visually assessed stenosis severity defined by coronary CTA showed a poor correlation with the functional significance determined by FFR [12, 13]. Validated by FFR, the quantitative plaque features improved the identification of lesion-specific ischemia when compared with visually evaluated stenosis grading. A total PB of $42 \%$ resulted in a specificity of $74.3 \%$ for the identification of hemodynamically relevant coronary artery stenosis, while qualitative diameter stenosis over 50\% lumen reduction resulted in a specificity of only $37.1 \%(P=0.0033)[14]$. The quantitative assessment of plaques with advanced characteristics provided incremental value to mere stenosis grading [15].

It is important to note that only a minority of myocardial infarctions is caused by significant stenosis, and approximately $50 \%$ of the cases plaque ruptures involve lesion sites without significant stenosis [16-21]. Furthermore, nonobstructive lesions with the presence of plaques with advanced characteristic features prone to cause most coronary events $[22,23]$. This means that $\mathrm{PB}$ quantification and plaque morphology evaluation are likely as important as assessing diameter stenosis alone. A shift in focus is needed in order to improve overall clinical outcome of the diagnosis and treatment of CAD.

Adverse plaque features are positive remodeling, spotty calcification, low attenuation and the napkin-ring sign (NRS). Plaques with adverse features are specific indicators of vulnerable plaques, therefore they allow a timely identification of patients at risk of future myocardial infarction [24]. According to Motoyama et al. [25] adverse plaque was found to be an independent parameter for the prediction of ACS. The PROMISE (Prospective Multicenter Imaging Study for Evaluation of Chest Pain) trial showed that the chance of future major adverse cardiac event (MACE) increases by $70 \%$ if an adverse plaque is present in the coronary arteries, regardless of the severity of the obstruction and clinical risk scores [26].

A quantitative study of low-to-intermediate CAD risk patients, reported that among adverse plaque features, the NRS (hazard risk:3.85; 95\% CI:1.7-8.6 $(P<0.0001)$ ) and lowattenuation plaque volume $(\leq 60$ Hounsfield unit $(\mathrm{HU}))$ (hazard risk:4.96; 95\% CI:2.0-12.2 $(P<0.0001)$ ) were the most powerful MACE predictors, after a mean follow-up time of 7.8 years [27]. In a study of 1,168 consecutive patients with suspected CAD, quantitative semi-automated plaque evaluation was performed in order to analyze low-attenuation plaque volume $(<30 \mathrm{HU})$, total non-calcified plaque volume $(<150 \mathrm{HU})$, remodeling index (the ratio of the coronary cross-sections of lumen area at the site of maximal stenosis and at the reference segment $[28,29])$ and the presence of plaques with NRS. Quantitatively identifying advanced plaque characteristics such as total PV and adverse plaque features improved prediction of MACE independently and incrementally of conventional coronary CTA risk scoring and calcium scoring after a mean follow-up of 5.7 years [30]. 
Ferencik et al. [31] defined the ROMICAT (Rule Out Myocardial Infarction/Ischemia Using Computer Assisted Tomography) score as the number of adverse plaque feature types from the ROMICAT I study. Applying it to the ROMICAT II study has proven the score to be an independent factor for the prediction of ACS. A retrospective qualitative study reported by Puchner et al. [32] on the ROMICAT II trial proved that having an adverse plaque in coronary arteries, regardless of cardiovascular factors and significant CAD, increases the chances of future ACS (odds ratio:8.9; 95\% CI:1.8-43.3 $(P=0.006)$ ). Both quantitative and qualitative readings of plaques with adverse features from the ROMICAT II study turned out to have good utility in predicting the increased likelihood for future ACS events $[31,32]$. That raises the question whether there is an incremental value of quantitative adverse plaque characterization over the much faster qualitative assessment.

The presence of adverse plaque features increased the occurrence of coronary heart disease resulting in either death or nonfatal myocardial infarction by 3 times during the 5-year long follow-up of the SCOT-HEART (Scottish Computed Tomography of the HEART) trial [24]. The SCOT-HEART trial found low attenuation PB to have the greatest power out of conventional and quantitative plaque features for predicting future myocardial infarctions in potential clinical usage of plaque type quantification. Specific finding was a probability increase of $400 \%$ for subsequent myocardial infarction if low attenuation PB was above $4 \%$ of all quantified plaque types (hazard ratio:4.65; 95\% CI:2.06$10.5(P<0.001))[33]$.

\section{Types of plaque quantification methods}

\section{Semi-automated plaque quantification}

Semi-automated methods are currently applied in scientific research, which make accurate quantification of $\mathrm{PB}$ possible. The use of a semi-automated approach instead of manual measurements improves reproducibility and reduces the difference between experienced and inexperienced readers [34]. One of the possible explanations to this difference might be that while display window settings affect manual measurements, semi-automated methods are immune to them [35].

Plaque quantification is currently carried out in several steps. As a first step, the reader marks the vessel of interest. Depending on the analysis software, this may only require the marking of the proximal and distal points, or it may be necessary to place markers along the vessel's centerline. One widely used automatic centerline marking method is described by Yang et al. [36]. In the second step, the software transforms curved vessels into straight ones (stretched multiplanar-reformatted volume). Then it calculates longitudinal cross-sections, usually 4 images, extracted at $45^{\circ}$ intervals and then at $0.5 \mathrm{~mm}$ intervals cross-sectional images are extracted based on the longitudinal borders of the vessel. The software then automatically tries to establish vessel borders, based on luminal intensity values by a model-guided minimum cost approach. Vessel parts with diameter less than $2 \mathrm{~mm}$ are excluded. The inner and outer vessel walls are subsequently presented to the reader for manual correction. Usually a so-called no-gap method is applied, assuming that the gap between the inner and outer vessel walls is only the plaque itself. Plaque types can be characterized by $\mathrm{HU}$ density values [37, 38]. This whole process, while already substantially automated, is still labor intensive. For experienced readers, it may take up to $30 \mathrm{~min}$ per vessel to adjust borders manually [8].

While it may be very hard (currently impossible) to give an analytical 'formula' for vessel boundary extraction, progress in computer science surprisingly makes it possible to utilize human expertise in automated image processing. This is the territory of deep learning. Nowadays one can feed data (images) annotated by human experts to the computer which subsequently 'learns' how to characterize images by itself. The pre-annotated data is called the 'training set'. There is an abundance of deep learning algorithms. Ones using various forms of neural network simulations are typical. In a study using a convolutional neural network for extracting centerlines, a 93.7\% overlap was achieved over manually marked reference centerlines [39].

\section{Automated plaque quantification}

Controversial results can be found in the literature concerning the reproducibility of fully automated plaque quantification methods. The study of Kolossvary et al. [40] found that the automated segmentation method compared with expert readers had poor agreement regarding different quantitative plaque features with the exception of calcified plaques. On the contrary, an IVUS validated dedicated automated quantitative software analysis performed with excellent reproducibility in determining quantitative plaque parameters with intraclass correlation coefficient (ICC) of 0.880 for PB [41]. Nevertheless, manual steps are required even with automated algorithms. Marking the starting point and the end of plaque lesions can lead to inaccurate PV quantification due to the involvement of visual recognition. In order to reduce the impact of visual adjustment, a voxelmap quantitative analysis approach has been developed. A three-dimensional coronary artery wall model is used to quantitatively and structurally analyze coronary artery walls, resulting in a similar approach as IVUS [42]. Nevertheless, these methods are still in an experimental stage. Generally, software tends to overestimate the size of calcified lesions and underestimate size of non-calcified plaques or even leave them unrecognized [43].

\section{Reproducibility of quantitative metrics}

\section{Intra- and inter-scanner reproducibility}

The intra-scanner variability for the Aquilion One Vision; Toshiba, Otawara, Japan device was found to be $\pm 18.4 \%$ (ICC of 0.948), however the inter-scanner variability for the above device and the Somatom Force; Siemens, Forchheim, 
Germany rose to $\pm 29.9 \%$ (ICC of 0.609 ) [44]. Seemingly, the reproducibility of plaque quantification might be variable with different computed tomography scanners.

\section{Intra- and inter-platform reproducibility}

Many commercially available image analysis software platforms exist. However, while these software tools have good intra-platform reproducibility, inter-platform reproducibility is poor [45]. Therefore, serial or comparative clinical studies require the use of the same software.

\section{Intra- and inter-observer reproducibility}

When using a single platform, intra- and inter-observer variability of semi-automated quantitative plaque evaluation should be reduced to a minimum. Thus, broad clinical application of quantitative plaque analysis would be feasible. Within and between observers, the geometrical and compositional assessment of coronary plaques is highly reproducible, although the inter-observer variability for compositional assessment has greater variance. However, one the most relevant elements in the clinical approach, the low attenuation $(<30 \mathrm{HU})$ plaque assessment had the highest inter-observer variability $[46,47]$. Measuring reproducibility on 39 patients' data, plaques volume, mean attenuation values and remodeling index showed excellent intra- and inter-observer variability. All concordance correlation coefficients values for both intra- and inter-observer variability ranged between 0.85 and 0.98 [48].

Inter-observer agreement for adverse plaque features was fair (Fleiss' Kappa: $\boldsymbol{\kappa}=0.400$ ), although the concordance to individual adverse plaque features was found to be only poor-to-fair [49]. Among the adverse plaque features, the NRS revealed the lowest agreement (Fleiss' Kappa: $\boldsymbol{\kappa}=$ 0.148 ). Low concordance could be explained by the fact that the NRS is a qualitative feature, therefore its diagnosis relies on the reader's experience, with an observed propensity for over- or under-diagnosis [49-52].

\section{Quantitative assessment of non-calcified plaques}

Exact distinction of the vessel lumen, vessel wall, plaque and the surrounding tissues is the main challenge for plaque identification, quantification, and characterization. Proper identification of lipid rich plaques is crucial due to their strong prognostic value of future adverse cardiac events [33]. Identification of these plaques is based on computed tomography attenuation value differences. However, previous studies have used different density cut-off values to differentiate between various plaque components, therefore a reliable and uniform density cut-off values are warranted.

Establishing HU based cut-off values aids plaque characterization, although non-calcified plaques do not differ considerably in their chemical composition from the vessel wall or from the epicardial fat.
One of the main challenges of HU threshold-based plaque characterization is that luminal contrast enhancement and intracoronary attenuation has a strong impact on plaque attenuation values. Therefore, relying merely on mean attenuation measurements in plaque classification tends to convey inconsistent results [53-55].

Several studies tried to determine a simple HU threshold value for the identification and quantitative assessment of non-calcified plaques [56-58]. These studies used different modalities for validation. In a histological analysis of 8 postmortem hearts of patients with sudden coronary death, $<75$ HU cut-off value had the highest correlation coefficient [56]. When optical coherence tomography or IVUS were used for validation, these thresholds decreased to 60 and $30 \mathrm{HUs}$, respectively [57, 58].

Graaf and his colleagues [59] used automated software tools to quantify and characterize plaques on coronary CTA showing excellent correlation with IVUS. They employed two different methods for plaque classification. In the first approach the plaques were divided into 4 different plaque categories using a set of previously described cut-off values: necrotic core (correlations were observed: $r=0.523(P<$ $0.001))$, dense calcium $(r=0.736, P<0.001)$, fibrotic $(r=$ $0.695(P<0.001))$ and fibro-fatty tissue $(r=0.714(P<$ $0.001))[59,60]$. In the second method, HU thresholds were dynamically adapted based on lumen attenuation values. Both approaches correlated well with IVUS. For plaque type volume quantification, the dynamic threshold was found to be superior over the fixed threshold approach [59]. The usage of adaptive methods has its advantages by compensating for the modifying effects of image acquisition and reconstruction, which influence attenuation values. These effects may be, for example, the variability in slice thickness, in tube voltage settings, the difference between filters and imaging algorithms employed, or even simple measurement noise [21, 53, 55, 61-63].

\section{Conclusion and future perspectives}

Coronary CTA is a well-established modality for the detection of coronary artery stenosis. Coronary CTA allows not only precise visualization of the coronary lumen, but detailed imaging of the vessel wall. Prior studies have shown that the majority of myocardial infarctions originate from non-obstructive lesions. In these cases, detailed description of adverse plaque features is essential for the prevention of myocardial infarction. Therefore, a shift in focus to volumetric coronary plaque assessment should be considered, and future research is needed to clarify if treatment strategies considering coronary $\mathrm{PB}$ would improve outcomes.

However, in these days, reproducibility is a major problem. Since inter-platform reproducibility is poor, serial or comparative assessments require evaluation with the same software, while development of industry standards could improve inter-scanner reproducibility [45, 51]. Moreover, a consensus in the establishment of cut-off values is lacking. The wild scattering of $\mathrm{HU}$ thresholds likely 
signifies the limits of this method in the identification of low-attenuation plaques. Furthermore, a great number of different plaque types is used in research with minor or no differences generating a rather complex naming system.

Even though modern techniques like radiomics and machine learning showed superiority to conventional quantitative computed tomographic metrics, we have to take extreme care with these novel methods $[5,6,64,65]$. Since these methods can produce an enormous amount of data, which is very difficult to properly interpret, although it has the potential to produce metrics that are difficult or downright impossible to discover by visual (regardless of the level of expertise) and conventional quantitative assessment.

Our review suggests that quantitative plaque assessment has the potential to help guide management of patients with CAD and can potentially be used as a tool in determining hemodynamically significant lesions. However, further technical improvements are needed to increase reproducibility and reliability of these methods.

Funding: This study was supported by the National Research, Development and Innovation Office of Hungary (NKFIA; NVKP_16-1-2016-0017 National Heart Program).

Authors' contribution: All authors contributed significantly to the manuscript.

Conflict of interest: The authors have no conflict of interest to disclose.

\section{REFERENCES}

[1] Gouya H, Varenne O, Trinquart L, Touze E, Vignaux O, Spaulding C, et al.: Coronary artery stenosis in high-risk patients: 64-section CT and coronary angiography-prospective study and analysis of discordance. Radiology 2009; 252(2): 377-85.

[2] Knuuti J, Wijns W, Saraste A, Capodanno D, Barbato E, FunckBrentano C, et al.: 2019 ESC Guidelines for the diagnosis and management of chronic coronary syndromes: the task force for the diagnosis and management of chronic coronary syndromes of the European Society of Cardiology (ESC). Eur Heart J 2019; 41(3): 407-77.

[3] Matsumoto H, Watanabe S, Kyo E, Tsuji T, Ando Y, Otaki Y, et al.: Standardized volumetric plaque quantification and characterization from coronary CT angiography: a head-to-head comparison with invasive intravascular ultrasound. Eur Radiol 2019; 29: 6129-39.

[4] Dweck MR, Maurovich-Horvat P, Leiner T, Cosyns B, Fayad ZA, Gijsen FJH, et al.: Contemporary rationale for non-invasive imaging of adverse coronary plaque features to identify the vulnerable patient: a position Paper from the European society of cardiology working group on atherosclerosis and vascular biology and the European association of cardiovascular imaging. Eur Heart J Cardiovasc Imaging 2020; 21(11): 1177-83.

[5] Kolossvary M, Karady J, Szilveszter B, Kitslaar P, Hoffmann U, Merkely B, et al.: Radiomic features are superior to conventional quantitative computed tomographic metrics to identify coronary plaques with napkin-ring sign. Circ Cardiovasc Imaging 2017; 10(12).

[6] Kolossvary M, Karady J, Kikuchi Y, Ivanov A, Schlett CL, Lu MT, et al.: Radiomics versus visual and histogram-based assessment to identify atheromatous lesions at coronary CT angiography: an ex vivo study. Radiology 2019: 190407.

[7] Kolossváry M, Kellermayer M, Merkely B, Maurovich-Horvat P: Cardiac computed tomography radiomics: a comprehensive review on radiomic techniques. J Thorac Imaging 2018; 33(1): 26-34.

[8] Hell MM, Motwani M, Otaki Y, Cadet S, Gransar H, MirandaPeats R, et al.: Quantitative global plaque characteristics from coronary computed tomography angiography for the prediction of future cardiac mortality during long-term follow-up. Eur Heart J Cardiovasc Imaging 2017; 18(12): 1331-9.

[9] Lee SE, Sung JM, Rizvi A, Lin FY, Kumar A, Hadamitzky M, et al.: Quantification of coronary atherosclerosis in the assessment of coronary artery disease. Circ Cardiovasc Imaging 2018; 11(7): e007562.

[10] Versteylen MO, Kietselaer BL, Dagnelie PC, Joosen IA, Dedic A, Raaijmakers RH, et al.: Additive value of semiautomated quantification of coronary artery disease using cardiac computed tomographic angiography to predict future acute coronary syndrome. J Am Coll Cardiol 2013; 61(22): 2296-305.

[11] Stuijfzand W, van Rosendael A, Lin F, Chang H, van den Hoogen I, Gianni U, et al.: Stress myocardial perfusion imaging vs coronary computed tomographic angiography for diagnosis of invasive vesselspecific coronary physiology: predictive modeling results from the computed tomographic evaluation of atherosclerotic determinants of myocardial ischemia (CREDENCE) trial. JAMA Cardiol 2020; 5(12): 1338-48.

[12] Sarno G, Decraemer I, Vanhoenacker PK, De Bruyne B, Hamilos $\mathrm{M}$, Cuisset $\mathrm{T}$, et al.: On the inappropriateness of noninvasive multidetector computed tomography coronary angiography to trigger coronary revascularization: a comparison with invasive angiography. JACC Cardiovasc Interv 2009; 2(6): 550-7.

[13] Meijboom WB, Van Mieghem CA, van Pelt N, Weustink A, Pugliese F, Mollet NR, et al.: Comprehensive assessment of coronary artery stenoses: computed tomography coronary angiography versus conventional coronary angiography and correlation with fractional flow reserve in patients with stable angina. J Am Coll Cardiol 2008; 52(8): 636-43.

[14] Diaz-Zamudio M, Dey D, Schuhbaeck A, Nakazato R, Gransar H, Slomka PJ, et al.: Automated quantitative plaque burden from coronary CT angiography noninvasively predicts hemodynamic significance by using fractional flow reserve in intermediate coronary lesions. Radiology 2015; 276(2): 408-15.

[15] Park HB, Heo R, ÓH B, Cho I, Gransar H, Nakazato R, et al.: Atherosclerotic plaque characteristics by $\mathrm{CT}$ angiography identify coronary lesions that cause ischemia: a direct comparison to fractional flow reserve. JACC Cardiovasc Imaging 2015; 8(1): 1-10.

[16] Ambrose JA, Tannenbaum MA, Alexopoulos D, HjemdahlMonsen CE, Leavy J, Weiss M, et al.: Angiographic progression of coronary artery disease and the development of myocardial infarction. J Am Coll Cardiol 1988; 12(1): 56-62.

[17] Farb A, Burke AP, Tang AL, Liang TY, Mannan P, Smialek J, et al.: Coronary plaque erosion without rupture into a lipid core. A frequent cause of coronary thrombosis in sudden coronary death. Circulation 1996; 93(7): 1354-63. 
[18] Glaser R, Selzer F, Faxon DP, Laskey WK, Cohen HA, Slater J, et al.: Clinical progression of incidental, asymptomatic lesions discovered during culprit vessel coronary intervention. Circulation 2005; 111(2): 143-9.

[19] Min JK, Shaw LJ, Devereux RB, Okin PM, Weinsaft JW, Russo DJ, et al.: Prognostic value of multidetector coronary computed tomographic angiography for prediction of all-cause mortality. J Am Coll Cardiol 2007; 50(12): 1161-70.

[20] Falk E, Shah PK, Fuster V: Coronary plaque disruption. Circulation 1995; 92(3): 657-71.

[21] Kolossvary M, Szilveszter B, Merkely B, Maurovich-Horvat P: Plaque imaging with CT-a comprehensive review on coronary CT angiography based risk assessment. Cardiovasc Diagn Ther 2017; 7(5): 489-506.

[22] Chang HJ, Lin FY, Lee SE, Andreini D, Bax J, Cademartiri F, et al.: Coronary atherosclerotic precursors of acute coronary syndromes. J Am Coll Cardiol 2018; 71(22): 2511-22.

[23] Maddox TM, Stanislawski MA, Grunwald GK, Bradley SM, Ho PM, Tsai TT, et al.: Nonobstructive coronary artery disease and risk of myocardial infarction. Jama 2014; 312(17): 1754-63.

[24] Williams MC, Moss AJ, Dweck M, Adamson PD, Alam S, Hunter A, et al.: Coronary artery plaque characteristics associated with adverse outcomes in the SCOT-HEART study. J Am Coll Cardiol 2019; 73(3): 291-301.

[25] Motoyama S, Ito H, Sarai M, Kondo T, Kawai H, Nagahara Y, et al.: Plaque characterization by coronary computed tomography angiography and the likelihood of acute coronary events in midterm follow-up. J Am Coll Cardiol 2015; 66(4): 337-46.

[26] Ferencik M, Mayrhofer T, Bittner DO, Emami H, Puchner SB, Lu $\mathrm{MT}$, et al.: Use of high-risk coronary atherosclerotic plaque detection for risk stratification of patients with stable chest pain: a secondary analysis of the promise randomized clinical trial. JAMA Cardiol 2018; 3(2): 144-52.

[27] Feuchtner G, Kerber J, Burghard P, Dichtl W, Friedrich G, Bonaros $\mathrm{N}$, et al.: The high-risk criteria low-attenuation plaque $<60 \mathrm{HU}$ and the napkin-ring sign are the most powerful predictors of MACE: a long-term follow-up study. Eur Heart J Cardiovasc Imaging 2017; 18(7): 772-9.

[28] Achenbach S, Ropers D, Hoffmann U, MacNeill B, Baum U, Pohle $\mathrm{K}$, et al.: Assessment of coronary remodeling in stenotic and nonstenotic coronary atherosclerotic lesions by multidetector spiral computed tomography. J Am Coll Cardiol 2004; 43(5): 842-7.

[29] Mintz GS, Nissen SE, Anderson WD, Bailey SR, Erbel R, Fitzgerald PJ, et al.: American college of cardiology clinical expert consensus document on standards for acquisition, measurement and reporting of intravascular ultrasound studies (IVUS). A report of the American college of cardiology task force on clinical expert consensus documents. J Am Coll Cardiol 2001; 37(5): 1478-92.

[30] Nadjiri J, Hausleiter J, Jahnichen C, Will A, Hendrich E, Martinoff $S$, et al.: Incremental prognostic value of quantitative plaque assessment in coronary CT angiography during 5 years of follow up. J Cardiovasc Comput Tomogr 2016; 10(2): 97-104.

[31] Ferencik M, Mayrhofer T, Puchner SB, Lu MT, Maurovich-Horvat $\mathrm{P}$, Liu T, et al.: Computed tomography-based high-risk coronary plaque score to predict acute coronary syndrome among patients with acute chest pain - results from the ROMICAT II trial. J Cardiovasc Computed Tomography 2015; 9(6): 538-45.
[32] Puchner SB, Liu T, Mayrhofer T, Truong QA, Lee H, Fleg JL, et al.: High-risk plaque detected on coronary CT angiography predicts acute coronary syndromes independent of significant stenosis in acute chest pain: results from the ROMICAT-II trial. J Am Coll Cardiol 2014; 64(7): 684-92.

[33] Williams MC, Kwiecinski J, Doris M, McElhinney P, D’Souza MS, Cadet S, et al.: Low-attenuation noncalcified plaque on coronary computed tomography angiography predicts myocardial infarction: results from the multicenter SCOT-HEART trial (Scottish computed tomography of the HEART). Circulation 2020; 141(18): $1452-62$.

[34] Blackmon KN, Streck J, Thilo C, Bastarrika G, Costello P, Schoepf UJ: Reproducibility of automated noncalcified coronary artery plaque burden assessment at coronary CT angiography. J Thorac Imaging 2009; 24(2): 96-102.

[35] Maurovich-Horvat P, Ferencik M, Bamberg F, Hoffmann U: Methods of plaque quantification and characterization by cardiac computed tomography. J Cardiovasc Comput Tomogr 2009; 3(Suppl 2): S91-8.

[36] Yang G, Kitslaar P, Frenay M, Broersen A, Boogers MJ, Bax JJ, et al.: Automatic centerline extraction of coronary arteries in coronary computed tomographic angiography. Int J Cardiovasc Imaging 2012; 28(4): 921-33.

[37] Lee SE, Chang HJ, Rizvi A, Hadamitzky M, Kim YJ, Conte E, et al.: Rationale and design of the Progression of AtheRosclerotic PlAque DetermIned by Computed TomoGraphic Angiography IMaging (PARADIGM) registry: a comprehensive exploration of plaque progression and its impact on clinical outcomes from a multicenter serial coronary computed tomographic angiography study. Am Heart J 2016; 182: 72-9.

[38] Nakazato R, Shalev A, Doh JH, Koo BK, Gransar H, Gomez MJ, et al.: Aggregate plaque volume by coronary computed tomography angiography is superior and incremental to luminal narrowing for diagnosis of ischemic lesions of intermediate stenosis severity. J Am Coll Cardiol 2013; 62(5): 460-7.

[39] Wolterink JM, van Hamersvelt RW, Viergever MA, Leiner T, Isgum I: Coronary artery centerline extraction in cardiac CT angiography using a CNN-based orientation classifier. Med Image Anal 2019; 51: 46-60.

[40] Kolossváry M, Jávorszky N, Karády J, Vecsey-Nagy M, Dávid T, Simon J, et al.: Effect of vessel wall segmentation on volumetric and radiomic parameters of coronary plaques with adverse characteristics. J Cardiovasc Comput Tomogr 2021; 15(2): 137-45.

[41] Boogers MJ, Broersen A, van Velzen JE, de Graaf FR, El-Naggar $\mathrm{HM}$, Kitslaar $\mathrm{PH}$, et al.: Automated quantification of coronary plaque with computed tomography: comparison with intravascular ultrasound using a dedicated registration algorithm for fusion-based quantification. Eur Heart J 2012; 33(8): 1007-16.

[42] Li Y, Chen W, Liu K, Wu Y, Chen Y, Chu C, et al.: A voxel-map quantitative analysis approach for atherosclerotic noncalcified plaques of the coronary artery tree. Comput Math Methods Med 2013; 2013: 957195.

[43] Leber AW, Becker A, Knez A, von Ziegler F, Sirol M, Nikolaou K, et al.: Accuracy of 64-slice computed tomography to classify and quantify plaque volumes in the proximal coronary system: a comparative study using intravascular ultrasound. J Am Coll Cardiol 2006; 47(3): 672-7. 
[44] Symons R, Morris JZ, Wu CO, Pourmorteza A, Ahlman MA, Lima JA, et al.: Coronary CT angiography: variability of CT scanners and readers in measurement of plaque volume. Radiology 2016; 281(3): 737-48.

[45] Oberoi S, Meinel FG, Schoepf UJ, Nance JW, De Cecco CN, Gebregziabher M, et al.: Reproducibility of noncalcified coronary artery plaque burden quantification from coronary CT angiography across different image analysis platforms. AJR Am J Roentgenol 2014; 202(1): W43-9.

[46] Papadopoulou SL, Garcia-Garcia HM, Rossi A, Girasis C, Dharampal AS, Kitslaar PH, et al.: Reproducibility of computed tomography angiography data analysis using semiautomated plaque quantification software: implications for the design of longitudinal studies. Int J Cardiovasc Imaging 2013; 29(5): 1095-104.

[47] Klass O, Kleinhans S, Walker MJ, Olszewski M, Feuerlein S, Juchems M, et al.: Coronary plaque imaging with 256-slice multidetector computed tomography: interobserver variability of volumetric lesion parameters with semiautomatic plaque analysis software. Int J Cardiovasc Imaging 2010; 26(6): 711-20.

[48] Lee MS, Chun EJ, Kim KJ, Kim JA, Vembar M, Choi SI: Reproducibility in the assessment of noncalcified coronary plaque with 256-slice multi-detector CT and automated plaque analysis software. Int J Cardiovasc Imaging 2010; 26(Suppl 2): 237-44.

[49] Maroules CD, Hamilton-Craig C, Branch K, Lee J, Cury RC, Maurovich-Horvat $\mathrm{P}$, et al.: Coronary artery disease reporting and data system (CAD-RADS(TM)): inter-observer agreement for assessment categories and modifiers. J Cardiovasc Comput Tomogr 2018; 12(2): 125-30.

[50] Maurovich-Horvat P, Hoffmann U, Vorpahl M, Nakano M, Virmani R, Alkadhi H: The napkin-ring sign: CT signature of high-risk coronary plaques? JACC Cardiovasc Imaging 2010; 3(4): 440-4.

[51] Maurovich-Horvat P, Ferencik M, Voros S, Merkely B, Hoffmann $\mathrm{U}$ : Comprehensive plaque assessment by coronary $\mathrm{CT}$ angiography. Nat Rev Cardiol 2014; 11(7): 390-402.

[52] Maurovich-Horvat P, Schlett CL, Alkadhi H, Nakano M, Otsuka F, Stolzmann P, et al.: The napkin-ring sign indicates advanced atherosclerotic lesions in coronary CT angiography. JACC Cardiovasc Imaging 2012; 5(12): 1243-52.

[53] Dalager MG, Bottcher M, Dalager S, Andersen G, Thygesen J, Pedersen EM, et al.: Imaging atherosclerotic plaques by cardiac computed tomography in vitro: impact of contrast type and acquisition protocol. Invest Radiol 2011; 46(12): 790-5.

[54] Dalager MG, Bottcher M, Andersen G, Thygesen J, Pedersen EM, Dejbjerg L, et al.: Impact of luminal density on plaque classification by CT coronary angiography. Int J Cardiovasc Imaging 2011; 27(4): 593-600.

[55] Cademartiri F, Mollet NR, Runza G, Bruining N, Hamers R, Somers $\mathrm{P}$, et al.: Influence of intracoronary attenuation on coronary plaque measurements using multislice computed tomography: observations in an ex vivo model of coronary computed tomography angiography. Eur Radiol 2005; 15(7): 1426-31.

[56] Han D, Torii S, Yahagi K, Lin FY, Lee JH, Rizvi A, et al.: Quantitative measurement of lipid rich plaque by coronary computed tomography angiography: a correlation of histology in sudden cardiac death. Atherosclerosis 2018; 275: 426-33.

[57] Marwan M, Taher MA, El Meniawy K, Awadallah H, Pflederer T, Schuhback A, et al.: In vivo $\mathrm{CT}$ detection of lipid-rich coronary artery atherosclerotic plaques using quantitative histogram analysis: a head to head comparison with IVUS. Atherosclerosis 2011; 215(1): 110-5.

[58] Schlett CL, Maurovich-Horvat P, Ferencik M, Alkadhi H, Stolzmann P, Scheffel H, et al.: Histogram analysis of lipid-core plaques in coronary computed tomographic angiography: ex vivo validation against histology. Invest Radiol 2013; 48(9): 646-53.

[59] de Graaf MA, Broersen A, Kitslaar PH, Roos CJ, Dijkstra J, Lelieveldt BP, et al.: Automatic quantification and characterization of coronary atherosclerosis with computed tomography coronary angiography: cross-correlation with intravascular ultrasound virtual histology. Int J Cardiovasc Imaging 2013; 29(5): 1177-90.

[60] Brodoefel H, Reimann A, Heuschmid M, Tsiflikas I, Kopp AF, Schroeder S, et al.: Characterization of coronary atherosclerosis by dual-source computed tomography and HU-based color mapping: a pilot study. Eur Radiol 2008; 18(11): 2466-74.

[61] Achenbach S, Boehmer K, Pflederer T, Ropers D, Seltmann M, Lell $\mathrm{M}$, et al.: Influence of slice thickness and reconstruction kernel on the computed tomographic attenuation of coronary atherosclerotic plaque. J Cardiovasc Comput Tomogr 2010; 4(2): $110-5$.

[62] Cademartiri F, La Grutta L, Runza G, Palumbo A, Maffei E, Mollet $\mathrm{NR}$, et al.: Influence of convolution filtering on coronary plaque attenuation values: observations in an ex vivo model of multislice computed tomography coronary angiography. Eur Radiol 2007; 17(7): 1842-9.

[63] Tanami Y, Ikeda E, Jinzaki M, Satoh K, Nishiwaki Y, Yamada M, et al.: Computed tomographic attenuation value of coronary atherosclerotic plaques with different tube voltage: an ex vivo study. J Comput Assist Tomogr 2010; 34(1): 58-63.

[64] Kolossvary M, Park J, Bang J, Zhang, J, Lee J, Paeng, J, et al.: Identification of invasive and radionuclide imaging markers of coronary plaque vulnerability using radiomic analysis of coronary computed tomography angiography. Eur Heart J Cardiovasc Imaging 2019; 20(11): 1250-8.

[65] Masuda T, Nakaura T, Funama Y, Okimoto T, Sato T, Higaki T, et al.: Machine-learning integration of CT histogram analysis to evaluate the composition of atherosclerotic plaques: validation with IB-IVUS. J Cardiovasc Comput Tomogr 2019; 13(2): 163-9.

Open Access. This is an open-access article distributed under the terms of the Creative Commons Attribution 4.0 International License (https://creativecommons.org/ licenses/by/4.0/), which permits unrestricted use, distribution, and reproduction in any medium, provided the original author and source are credited, a link to the CC License is provided, and changes - if any - are indicated. (SID_1) 